Annuaire suisse de politique de développement

\title{
La société globale de l'information : visions, peuples et pouvoirs
}

Cees J. Hamelink

\section{(2) OpenEdition}

12 Journals

Édition électronique

URL : http://journals.openedition.org/aspd/580

ISSN : 1663-9669

\section{Éditeur}

Institut de hautes études internationales et du développement

Édition imprimée

Date de publication : 1 novembre 2003

Pagination : 159-183

ISSN : 1660-5934

Référence électronique

Cees J. Hamelink, «La société globale de l'information : visions, peuples et pouvoirs », Annuaire suisse de politique de développement [En ligne], 22-2 | 2003, mis en ligne le 22 mars 2010, consulté le 07 septembre 2020. URL : http://journals.openedition.org/aspd/580 


\title{
La société globale de l'information: visions, peuples et pouvoirs
}

\author{
Cees J. Hamelink*
}

\begin{abstract}
C et article analyse les conceptions de la société de l'information présentées dans un grand nombre de documents préparatoires du SMSI. Il conclut que ces textes sont empreints d'une tonalité d'optimisme technologique. L'un des éléments marquants dans ces textes est l'importance accordée à la notion d' "information», au détriment de la "communication», qui en est pour ainsi dire absente. C'est un fait préoccupant, dans la mesure où, pour créer une société mondiale ouverte à tous et pour permettre l'émancipation des populations, l'impératif le plus urgent est de permettre aux gens de communiquer.
\end{abstract}

Dans la majeure partie des documents préparatoires du SMSI, on constate aussi une absence préoccupante d'analyse sociale fondamentale. Il en résulte que la plupart des affirmations sont gratuites et ne reposent sur rien. L'absence de lien entre la "fracture numérique» et le phénomène planétaire qu'est la "fracture $d u$ développement» en est l'illustration. L'article propose une étude critique des textes juridiques en vigueur dans le domaine des télécommunications et des droits de propriété intellectuelle. Il décrit les mutations cruciales en cours dans la politique mondiale de l'information et de la communication et propose, dans l'optique du SMSI, une vision articulée sur l'être humain et sur les droits de l'homme. Cette conception exige la formation d'une coalition de parties prenantes soucieuses de créer une société de l'information façonnée à partir de la base. L'article se conclut par une liste de recommandations à l'intention d'un tel groupe.

Le Sommet mondial sur la société de l'information (SMSI) a été organisé pour débattre d'une notion controversée. Il n'existe pas de définition généralement reconnue de la société de l'information. Le sens même de cette expression a été fondamentalement mis en question; des articles universitaires ont été jusqu'à suggérer que la notion était sans rapport avec les réalités sociales actuelles. Des doutes ont par ailleurs été émis quant à la possibilité de décrire valablement des sociétés au moyen d'un seul critère, et même si cela était possible, on peut se demander si l'information est une catégorie plus précise que l'argent, la criminalité ou l'agression. En tout état de cause, il importe de relever que les sociétés se développent en suivant des parcours très différents, et si l'on veut vraiment faire référence à l'information, mieux vaudrait utiliser la notion plurielle de «sociétés de l'information». L'expression «société de l'information» peut signifier des choses fort différentes selon les gens: davantage de téléphones, plus d'argent, davantage de réglementation ou plus d'émancipation.

* Professeur en communication internationale à l'Université d'Amsterdam. Hollande. 
Il est très malheureux que dans les documents préparatoires du sommet, la notion de société de l'information elle-même soit adoptée sans le moindre regard critique. On retrouve couramment une formulation qui figure dans la contribution de l'Union européenne à la deuxième réunion du Comité de préparation (organisée en février 2003 à Genève), à savoir que la société de l'information «offre de grandes possibilités de promouvoir le développement durable, la démocratie, la transparence, la responsabilité et la bonne gouvernance». Il est pour le moins embarrassant de voir une organisation sérieuse affirmer qu'une entité qu'elle ne prend pas la peine de définir (la société de l'information) pourrait réaliser quelque chose. Cela dit, certains des textes préparatoires tentent de définir la société de l'information.

Ainsi, la Déclaration de Bávaro, adoptée par la Conférence ministérielle régionale de préparation du SMSI pour l'Amérique latine et les Caraïbes (19-31 janvier 2003), affirme que «la société de l'information est un système économique et social dans lequel la connaissance et l'information constituent les sources fondamentales du bien-être et du progrès ». La Déclaration de Tokyo de la Conférence régionale de préparation du SMSI pour l'Asie-Pacifique (13-15 janvier 2003) affirme, quant à elle, que la société de l'information est «une société dans laquelle les réseaux d'information et de communication très développés, l'accès équitable et généralisé à l'information, l'existence d'un contenu adapté et facilement accessible et l'efficacité de la communication peuvent aider les êtres humains à réaliser leur potentiel, promouvoir un développement socio-économique durable, améliorer la qualité de la vie, lutter contre la pauvreté et la faim, et faciliter la prise de décision collective».

\section{Des visions positives, voire euphoriques}

Les conceptions de la société de l'information présentées par les diverses parties prenantes sont la plupart du temps réconfortantes et optimistes. On retrouve les mêmes mots clés dans la majorité des textes. La société de l'information devrait être ouverte à tous et permettre la participation et l'accès les plus larges possibles. La société de l'information devrait contribuer à l'émancipation des populations, créer un environnement propice et soutenir le renforcement des capacités. La société de l'information devrait être dirigée démocratiquement. Les objectifs premiers sont le développement durable, la diversité culturelle et l'attention à la problématique hommes-femmes.

Le projet de déclaration finale (texte daté du 25 février 2003) contient des phrases caractéristiques à cet égard :

$\square$ En mobilisant le potentiel qu'offrent les technologies de l'information et de la communication (TIC), nous pouvons trouver des réponses nouvelles et mieux adaptées à la lutte contre la pauvreté, l'équité et la justice sociale.

$\checkmark$ La connaissance et l'information sont la source fondamentale du bien-être et du progrès.

$\square$ L'immense potentiel qu'ont les TIC d'améliorer la productivité et la qualité de la vie est une grave question. 
$\square \quad$ Le Sommet, qui doit mettre l'accent sur le développement, doit avoir pour objectif d'apporter à tous les avantages de la société de l'information.

$\checkmark$ Dans l'édification d'une société de l'information, il convient de faire en sorte que les femmes bénéficient comme les hommes de l'utilisation accrue des TIC pour qu'elles s'émancipent et participent pleinement au développement politique, économique et social.

$\square$ La diversité culturelle et linguistique doit être encouragée et les capacités des populations autochtones devraient être employées pour contribuer à ce processus.

$\square \quad$ La société de l'information doit s'orienter vers l'élimination des différences socio-économiques existant au sein de nos sociétés.

$\square$ Un accès équitable et approprié pour tous est indispensable pour une infrastructure des réseaux d'information et de communication bien développée, économiquement abordable et facilement accessible.

$\square$ Fournir des services d'information et de communication ciblant les groupes sociaux défavorisés, en particulier les catégories à faible revenu, afin de contribuer à la lutte contre la pauvreté.

$\square$ Chacun devrait être en mesure d'acquérir les compétences nécessaires pour pouvoir jouer un rôle actif dans la société de l'information, en comprendre le fonctionnement, et tirer pleinement parti des possibilités qu'elle offre.

$\square$ Afin de pouvoir tirer parti au maximum des possibilités de la société de l'information, les pouvoirs publics doivent créer un cadre juridique, réglementaire et politique fiable, transparent et non discriminatoire.

$\square \quad$ La société de l'information est fondée sur le respect de l'expression culturelle et sur la possibilité qui est offerte d'en tirer pleinement parti.

$\square \quad$ Le plurilinguisme et le maintien de la diversité culturelle doivent être encouragés comme moteur des processus de développement de contenus à usages locaux et internationaux.

$\square \quad$ La participation pleine et efficace de toutes les parties prenantes est vitale pour mettre au point de nouvelles applications des TIC.

$\square$ La communauté internationale est appelée à fournir une coopération technique et financière sur les plans multilatéral et bilatéral.

L'impression générale qui ressort de ces affirmations est que la société de l'information pourrait créer une situation sans précédent, où chacun trouverait son compte, et contribuer à une vie meilleure pour tous. La plupart des participants au processus du Sommet mondial semblent adhérer à la vision proposée par le président du Comité de préparation du sommet, Adama Samassékou, exposée dans un document d'orientation présenté à la deuxième session du Comité en février à Genève, dans lequel il définit la société mondiale de l'information intégrée comme une société où «tout le monde, sans distinction, a les moyens de créer, de recevoir, de partager et d'utiliser librement informations et connaissances pour son épanouissement économique, social, culturel et politique».

Voilà des intentions louables, mais force est de constater qu'elles sont formulées sous forme de visions dépourvues de tout élément empirique qui pourrait 
étayer le point de vue selon lequel la société de l'information offrirait réellement ces possibilités.

\section{Le "technoptimisme»}

Il émane de presque tous les textes préparatoires une tonalité positive, voire euphorique, sur le potentiel des TIC. On trouve couramment des expressions telles que «les technologies de l'information et de la communication en tant qu'outil de développement peuvent conférer des avantages considérables». On ne peut manquer d'être frappé, dans la plupart des contributions, par l'optimisme que suscite la technique. Cette attitude est extrêmement préoccupante, car elle dénote un refus d'envisager sérieusement des scénarios négatifs quant à l'évolution future. Or, comme toutes les techniques, les TIC ont leurs inconvénients; dès lors, il serait tout simplement rationnel et responsable de se préparer au scénario le moins favorable.

La notion de société de l'information est ancrée dans la culture technologique contemporaine. La relation dominante de la société avec la technique est marquée dans une large mesure par l'irrationnel et l'irresponsabilité. On peut l'illustrer en faisant appel à trois figures symboliques: le Titanic, Cassandre et le docteur Frankenstein.

- Le Titanic représente une croyance profonde en la perfection de la technique: à quoi bon s'embarrasser d'un nombre de bouées suffisant puisque le navire ne peut pas sombrer? C'est ainsi que les risques réels des innovations techniques ne sont pas pris au sérieux. La culture technologique moderne tend fortement vers une société d'où tout risque est exclu. Ce désir de parvenir à une maîtrise des processus sociaux sans le moindre risque est gravement contrarié par le côté imprévisible et inconstant de l'espèce humaine. L'être humain, de plus en plus souvent, vient à être perçu comme le vrai facteur de risque. C'est la raison pour laquelle les sociétés modernes ne cessent de concevoir toutes sortes de mesures pour réduire les risques, comme la surveillance de plus en plus répandue au moyen de caméras omniprésentes, ou encore l'enregistrement électronique des déplacements des personnes. En toute logique, l'étape suivante dans ce processus devrait être le remplacement des êtres humains par des robots humanoïdes.

- Cassandre était la fille de Priam, le roi de Troie; c'est elle qui a prévenu les Troyens de la présence des Grecs dans le cheval de bois. Cassandre avait le don de prédire l'avenir, mais Apollon l'avait aussi affligée d'une malédiction: personne n'écouterait ses avertissements. C'est une caractéristique de la culture technologique: les avertissements ne sont pas entendus. Dans des situations où les décideurs ont le sentiment d'inaugurer une ère nouvelle, où l'humeur est à l'optimisme, et où chacun subit la pression du temps et de la concurrence, tous les signaux d'alerte seront négligés, les voix dissidentes étouffées, et le choix technologique devient un pilotage à l'aveugle.

$\square$ Le docteur Frankenstein est le héros du roman de Mary Shelley (1818) dans lequel le docteur qui a créé un monstre fuit son laboratoire, poursuivi par le monstre, qui le met en demeure d'assumer la responsabilité de sa création. 
La métaphore soulève la question essentielle de la responsabilité des innovations technologiques. Qui est responsable quand les choses dérapent? Qui assumera la responsabilité si, une fois comblée la fracture numérique, nous faisons face à d'insurmontables problèmes écologiques: niveaux beaucoup trop élevés de consommation d'énergie à l'échelle globale, taux d'émission de $\mathrm{CO}_{2}$ et volume croissant de déchets électroniques causés par le taux d'obsolescence rapide des téléphones portables et des ordinateurs?

\section{Et la communication?}

La notion de société de l'information repose - c'est une lapalissade - sur le concept d'information. La réflexion sur l'avenir des sociétés de l'information se fonde pour une bonne part sur une série de propositions qui sont autant de mythes populaires: l'abondance d'information est toujours une bonne chose; l'information est source de connaissance et de compréhension; des flux ouverts d'informations contribuent à la prévention des conflits; qui dit plus d'information dit moins d'incertitude et des choix plus pertinents; des personnes dûment informées agissent en conséquence; plus d'information égale davantage de pouvoir; une fois que les gens en sauront plus les uns sur les autres, ils se comprendront mieux et seront moins enclins au conflit. Autant d'hypothèses fort attrayantes... mais aucune d'entre elles n'est nécessairement vraie !

Pour résoudre les problèmes les plus urgents de la planète, les gens n'ont pas nécessairement besoin de davantage d'informations et de connaissances, mais bien plutôt d'acquérir la capacité de se parler, en surmontant les clivages imposés par la culture, la religion et la langue. Le dialogue est absolument crucial pour les contacts entre les civilisations. Il est déconcertant de constater que la notion de communication a pour ainsi dire disparu de la plupart des documents préparatoires pour le Sommet mondial sur la société de l'information. Le danger est bien réel de voir le sommet commettre la même erreur que la Conférence mondiale des Nations unies sur les droits de l'homme (Vienne, 1993), qui, dans sa déclaration finale, ne mentionnait pas la communication, mais uniquement l'information et les nouvelles. Or, l'enjeu fondamental est bien la création de «sociétés de la communication». Le monde n'a pas, en réalité, besoin d'une capacité accrue de traiter les informations, mais bien d'une capacité de communiquer. Ironie du sort: à mesure que se renforce et s'améliore notre capacité de traiter et de diffuser informations et connaissances, notre capacité de communication et d'échange s'atrophie.

Dans les sociétés complexes d'aujourd'hui, nous avons un besoin urgent de communiquer les uns avec les autres. Pour pouvoir faire face aux problèmes sociaux les plus pressants, la capacité de communiquer est bien plus cruciale que la capacité d'informer. Il est extrêmement préoccupant de constater que nous pourrions parvenir à bâtir des sociétés de l'information, voire de la connaissance, dans lesquelles les gens seraient incapables de se parler. Encore une fois, le fait que la communication ne soit pas mentionnée constitue une grave omission. Cette vision reflète l'idée simpliste selon laquelle la création, la réception, le partage et l'utilisation de l'information et des connaissances 
contribueront aux processus de développement, même si les gens n'ont pas la capacité ou la possibilité de communiquer.

Il est inquiétant de constater, dans les documents qui mentionnent la communication, une ignorance apparemment complète de la distinction fondamentale entre information et connaissances, d'une part, et communication, de l'autre. On a souvent l'impression d'un amalgame entre communication et diffusion d'idées et d'informations. C'est quelque peu compréhensible dans la mesure où ces mêmes documents font fréquemment référence aux médias comme à des moyens de communication. Certes, les médias peuvent remplir cette fonction, mais il n'empêche que la plupart du temps ils sont de simples moyens de diffusion. Les informations et les connaissances qu'ils diffusent peuvent mener à des processus interactifs, mais c'est loin d'être toujours le cas...

Pour créer une société globale ouverte à tous et donner à la population les outils nécessaires au développement économique, social, culturel et politique, la communication est l'un des enjeux les plus urgents. Or, on ne trouve nulle part (exception faite des positions défendues par la campagne Communication Rights in the Information Society - CRIS) de vision d'une société de l'information globale ouverte à tous. La déclaration finale des ONG d'Asie (Bangkok, 22-24 novembre 2002) affirme, entre autres principes, que les droits à la communication sont essentiels pour la démocratie et le développement humain, et signale qu'ils sont déjà mentionnés dans des dispositions telles que l'article 19 de la Déclaration universelle des droits de l'homme. Or, cette affirmation dénote un grave malentendu. L'article 19, en effet, concerne les droits à l'information, il consacre le droit à la liberté d'opinion et d'expression; il ne concerne pas la communication! La communication est un phénomène qui n'a rien à voir avec la formulation et la diffusion d'informations et d'opinions. Il n'existe pas encore de droit fermement consacré à un environnement qui favorise la communication entre êtres humains.

\section{L'analyse sociale critique n'est pas à l'ordre du jour}

Quelles que soient ses lacunes sur le plan intellectuel, la notion de «société de l'information» présente aussi certains avantages sur le plan analytique. La référence à la «société » appelle les bonnes vieilles interrogations sociologiques sur le pouvoir, le profit et la participation: qui en bénéficie, qui décide, qui participe et qui est responsable? Or, c'est précisément cette analyse qui est absente de la plupart des documents préparatoires. Voilà qui ne peut manquer d'inquiéter, car la plupart des propositions louables sur ce qu'est ou devrait être la société de l'information s'inscrivent dans un programme international pour un monde meilleur... qui a déjà bien servi. Aucun des mots d'ordre des décennies écoulées ne manque à l'appel: démocratie, diversité, capacités, participation, égalité des sexes et combler les fossés. Tant d'originalité laisse pantois! Une seule question se pose: pourquoi donc la communauté internationale n'a-t-elle pas, à ce jour, pris au sérieux ces aspirations? Pourquoi s'est-elle montrée si peu disposée, au cours des décennies écoulées, à se mettre concrètement à l'œuvre pour traduire en actes ses belles paroles? 
Avant de formuler toutes ces phrases gratuites sur le potentiel de la société de l'information, les TIC, l'information et la connaissance, il serait bon de poser quelques questions: pourquoi le monde n'est-il pas, aujourd'hui, une communauté ouverte à tous ? Pourquoi le développement durable est-il toujours une utopie? Pourquoi n'y a-t-il pas de gouvernance mondiale transparente et responsable? Pourquoi les citoyens ne peuvent-ils participer à la vie de leur société sur un pied d'égalité? La gestion de la société de l'information devrait susciter une discussion bien plus approfondie ! Là encore, les déclarations sonnent bien. Participation, parties prenantes, démocratie, transparence, voilà pour les slogans. Cela dit, pourquoi donc la gouvernance globale de la société de l'information devrait-elle se parer de toutes les caractéristiques qui sont jusqu'ici totalement absentes dans les autres domaines? Pourquoi devrait-on penser que des gouvernements qui ont si facilement eu recours à des notions telles que la «guerre contre le terrorisme» pour enfreindre les droits civils et politiques fondamentaux vont soudain se montrer garants d'une gouvernance «axée sur l'être humain»? Pourquoi de grandes sociétés d'envergure mondiale devraient-elles soudain désirer davantage de transparence et de responsabilité à l'égard du public ?

Comment la société mondiale de l'information pourrait-elle être gérée de manière ouverte et démocratique si les gouvernements ne sont pas disposés à ce que le SMSI soit géré de manière ouverte et démocratique? Les citoyens ne peuvent même pas participer sur un pied d'égalité à une conférence sur la société de l'information! Les gouvernements des Etats membres de l'ONU ont fait en sorte de garder la maîtrise du Sommet mondial et de ne pas partager avec les autres participants le pouvoir de prendre les décisions définitives concernant les résultats du SMSI.

\section{La fracture numérique et la fracture du développement}

Le vœu de réduire la fracture numérique est un bon exemple de vision dépourvue d'analyse. On constate une tendance à traiter cette question essentiellement comme une conséquence de la répartition - biaisée à l'échelle globale - des ressources en matière d'information et de communication. Cette manière de voir revient à isoler la fracture numérique du problème plus vaste que constitue la fracture du développement. La fracture numérique n'est en réalité que l'un des nombreux avatars de la répartition inégale des ressources - aussi bien matérielles qu'immatérielles - dans le monde, que ce soit à l'intérieur des sociétés ou entre elles.

Aussi longtemps que ce problème fondamental ne sera pas réglé - et il y faudrait une volonté politique qui fait actuellement défaut au sein de la communauté internationale -, les déclarations solennelles sur la «fracture numérique» resteront des écrans de fumée pratiques à des fins de diversion. La question de la fracture numérique illustre, au fond, le choix moral formulé de manière si pertinente par Richard Rorty, selon lequel «on ne peut envisager aucune application de la technique qui rendrait toutes les familles suffisamment riches pour fournir à leurs enfants quoi que ce soit de comparable aux possibilités qui sont considérées comme allant de soi dans les parties du monde fortunées. On ne peut davantage attendre des populations des démocraties industrialisées qu'elles 
redistribuent leurs richesses pour créer des perspectives prometteuses pour les enfants des pays sous-développés, d'une manière qui menacerait les perspectives de leurs propres enfants $»^{1}$. Et Rorty de conclure: «La seule manière dont les riches peuvent se considérer comme faisant partie de la même communauté morale que les pauvres serait un scénario porteur d'espoir pour les enfants des pauvres, mais qui ne menace pas celui de leurs propres enfants. $»^{2}$

En d'autres termes, les riches ne sont disposés à agir en accord avec des principes moraux comme la solidarité humaine qu'à la condition que leurs propres intérêts ne soient pas menacés par ces actes. Que les perspectives de leurs propres enfants soient en jeu, et le choix se fera contre les principes moraux. Aucun d'entre nous, habitants des régions riches du monde, ne peut échapper au défi de ce choix moral.

Les visions de la société de l'information qui sont avancées par les nantis de ce monde sont pour une bonne part le fruit d'aspirations morales méritoires, comme l'éradication de la pauvreté ou la réduction des inégalités de revenus. Il peut s'agir, pour une bonne part, de la rhétorique officielle des politiques de développement, mais aussi d'un désir sincère de responsables politiques et d'agents de terrain bien intentionnés. Dans un cas comme dans l'autre, le problème est que ces aspirations pour les pauvres ne peuvent se réaliser sans limiter singulièrement les perspectives des riches.

C'est donc l'exemple même d'un dilemme moral insoluble, puisque les contraintes écologiques de notre planète font que les perspectives des pauvres ne peuvent s'améliorer qu'aux dépens des perspectives des riches. Impossible d'améliorer les conditions de vie de la majorité des gens «défavorisés» dans le monde pour les porter au niveau des minorités privilégiées sans causer une catastrophe écologique sans précédent. Tous les efforts de développement se heurtent donc à un défi sans appel: on n'améliorera pas le sort des pauvres sans toucher aux privilèges des riches.

Relever ce défi exige que les institutions et les personnes sincèrement désireuses de trouver une solution sérieuse à la fracture du développement prennent parti contre l'ordre économique néolibéral dominant, qui appauvrit la majorité de la population du monde tout en créant une richesse sans précédent pour des élites. Les données ne manquent pas pour conclure que les inégalités de revenus se creusent aujourd'hui plus rapidement que jamais, partout dans le monde. En 1960, les $20 \%$ d'habitants les plus riches de la planète étaient 30 fois plus riches que les $20 \%$ les plus pauvres. En 1991, ce ratio avait passé à 61 contre un, et en 1994 atteignait 78 contre un. La part des $20 \%$ d'habitants les plus pauvres représentait, en 1997, 1,1\% de l'ensemble des revenus mondiaux (contre 2,3\% en 1960). En 1997, sur une population mondiale de 5,7 milliards de personnes, 1,3 milliard vivaient avec moins de 2 dollars par jour, tandis que les 358 milliardaires les plus riches se partageaient $45 \%$ du revenu total de l'humanité.

1 Rorty, R., «Putting Your Money Where Your Mouth Is », UNESCO Sources, n 79, May 1996, Paris, UNESCO Press.

2 Ibid. 
". UN MONDE PLUS JUSTE ... ...LUTTER CONTRE LA PAUVRETE...
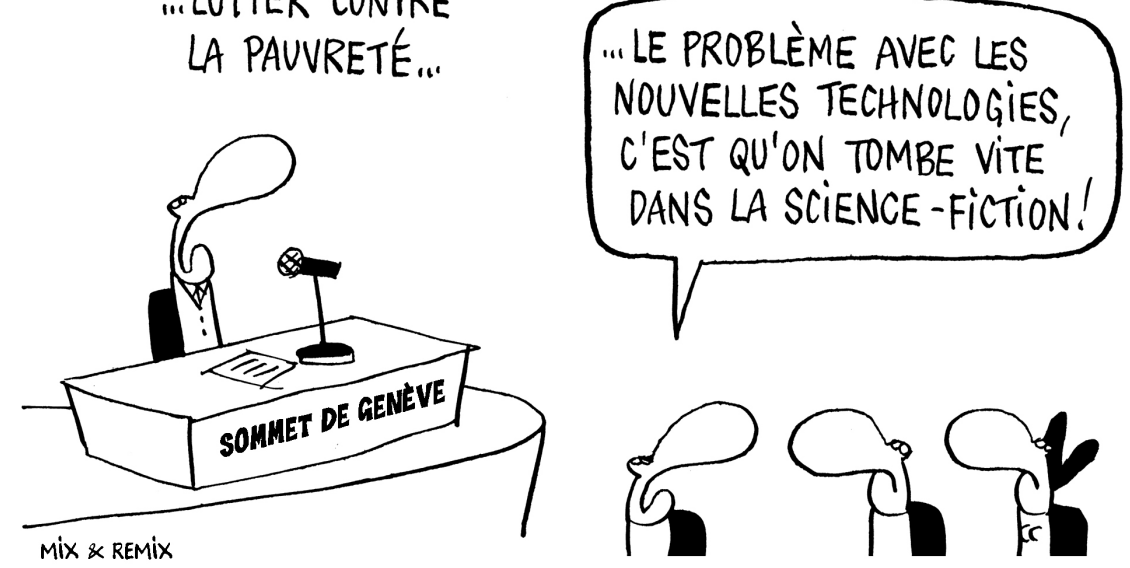

La pauvreté est l'une des caractéristiques de la mondialisation de l'économie, car elle ne touche plus uniquement les pays pauvres de l'hémisphère Sud. En Europe de l'Est, par exemple, le nombre de pauvres (disposant de moins de 4 dollars par jour pour vivre) dans les pays issus de l'ex-Union soviétique a passé de 14 millions en 1988 à 119 millions en 1994. En Europe occidentale, plus de 70 millions de pauvres sont menacés d'exclusion sociale.

On peut répondre à ces constatations peu réjouissantes que de nombreux pays ne connaissent qu'une croissance économique réduite. Il est indubitable que la pauvreté a peu de chances de disparaître en l'absence de croissance économique. Encore faut-il, pour réduire la pauvreté, que la croissance économique s'accompagne de programmes politiques ciblés pour soulager la pauvreté et de politiques publiques de redistribution des richesses. Qui plus est, l'expérience nous enseigne que lorsque la croissance économique réduit la pauvreté, cela ne signifie pas pour autant que les inégalités sociales soient réduites. Dans la plupart des pays, l'augmentation du produit intérieur brut bénéficie davantage aux riches qu'aux pauvres. Ainsi, au cours des dernières années, dans la plupart des pays d'Amérique latine, les revenus des $20 \%$ d'habitants les plus pauvres ont baissé. La distribution inégale des bénéfices économiques semble renforcée par le processus qu'on appelle mondialisation. La part des pays les moins avancés (qui regroupent $10 \%$ environ de la population mondiale) dans les échanges mondiaux a passé de 0,6\% dans les années 1970 à 0,3\% vingt ans plus tard. 
Il semble y avoir, dans la discussion actuelle concernant la fracture numérique, une hypothèse sans fondement selon laquelle les propriétaires des TIC modifieront leurs attitudes et leurs politiques à l'égard des transferts internationaux de technologies. Tout au long des décennies écoulées, les politiques internationales en matière de transfert de technologies ont créé des obstacles formidables à la réduction de la fracture technologique entre le Nord et le Sud.

Rien ne laisse présager aujourd'hui un changement radical dans les pratiques courantes de transfert de technologies. Il est, de ce fait, fort peu probable que les relations entre pays richement dotés en TIC et pays qui en sont privés se transforment dans un proche avenir. En outre, le partage équitable des infrastructures de communication (les systèmes d'autoroutes électroniques créés par les vecteurs de télécommunications tels que satellites, câbles, lignes fixes et transmissions mobiles), des capacités de calcul (ordinateurs, périphériques, réseaux), des ressources d'information (bases de données, bibliothèques) et des connaissances en matière de TIC (les capacités intellectuelles et sociales de déployer utilement les TIC) exigerait un effort gigantesque de la part de la communauté internationale. Des investissements considérables sont nécessaires pour rénover, mettre à niveau et étendre les réseaux dans les pays en développement, pour lancer des programmes de transfert de connaissances et pour assurer la formation aux TIC, en particulier pour les femmes.

En 1985, la Commission Maitland estimait à environ 12 milliards de dollars par an l'investissement nécessaire pour réaliser l'objectif, qu'elle appelait de ses vœux, de voir l'ensemble de la population mondiale avoir un accès aisé à un téléphone au début du XXI ${ }^{\mathrm{e}}$ siècle ${ }^{3}$. Le 8 décembre 1996, Gautan S. Kaji, directeur général de la Banque mondiale, déclarait dans un discours devant la Conférence ministérielle de Singapour de l'Organisation mondiale du commerce: «Nous estimons que les investissements dans les infrastructures de télécommunications dans les pays en développement, qui étaient en moyenne d'environ 30 milliards de dollars au cours de la période 1990-1994, devront doubler dans les cinq années à venir afin de mettre en œuvre les améliorations nécessaires. L'ampleur de ces investissements est manifestement supérieure aux capacités de financement découlant des recettes fiscales et des sources de financement publiques intérieures. Le secteur privé devra y mettre du sien. $»^{4}$

Peut-on raisonnablement compter sur le financement privé pour instaurer une équité mondiale en matière d'accès aux ressources des TIC et de leur utilisation? On peut se le demander. Il semblerait qu'en tout état de cause, la communauté internationale des gouvernements et les gouvernements nationaux des pays riches devraient se voir rappeler que ce n'est pas le manque de moyens financiers, mais bien le manque de volonté politique qui empêche de trouver des solutions. Instaurer un accès suffisant aux ressources des TIC dans le monde entier ne devrait pas poser de problème dans une économie mondiale qui dégage des revenus globaux de l'ordre de 22 billions de dollars. Le hic est que les

Commission Maitland, Le chaînon manquant, Genève, UIT, 1986.

I-Ways: Digest of the Global Information Infrastructure Commission, Washington, D.C., vol. 19, $\mathrm{n}^{\mathrm{o}} 2,1996, \mathrm{pp} .32-34$. 
dépenses en matière d'aide au développement ne représentent que 55 milliards de dollars, c'est-à-dire 0,25\% à peine de ce revenu. Comme le signalait en 1998 le Programme des Nations unies pour le développement (PNUD): «L'aide publique au développement est désormais au niveau le plus bas depuis que des statistiques existent. $»^{5}$

Pour estimer le montant des fonds nécessaires afin de fournir un accès universel à l'équipement et aux services de base pour les TIC, il faut tenir compte des coûts d'investissement pour les infrastructures essentielles et des coûts de fonctionnement réguliers. Poser un milliard de lignes téléphoniques, subventionner plus de 600 millions de ménages qui ne peuvent se permettre de payer des frais téléphoniques de base, fournir des ordinateurs et un accès à l'Internet pour les écoles: le coût pour l'ensemble des pays en développement pourrait s'élever à 80-100 milliards de dollars par an sur dix ans. Un tel niveau de financement ne devrait pas être insurmontable, puisqu'il représente environ $11 \%$ des dépenses militaires mondiales annuelles, ou environ $22 \%$ des dépenses mondiales annuelles en stupéfiants, ou encore le montant dépensé chaque année en boissons alcoolisées sur le seul continent européen ${ }^{6}$.

Pour toute une série de raisons politiques et économiques, de nombreux gouvernements de pays donateurs réduisent actuellement leur financement en faveur du développement des TIC. Entre 1990 et 1995, les crédits multilatéraux accordés à des projets de télécommunications ont passé de 1,253 milliard de dollars à 967 millions de dollars. L'aide bilatérale à des projets de télécommunications a passé de 1,259 milliard de dollars en 1990 à 800 millions de dollars en $1995^{7}$.

\section{Les dispositions juridiques essentielles}

Avant de promettre à la planète entière un effort sincère de réduire les inégalités actuelles en matière de disponibilité, d'accès et de coût de l'information, des infrastructures de communication et des contenus (nouvelles, informations générales, connaissances scientifiques, expressions artistiques), la communauté internationale devrait réviser l'ensemble des dispositions réglementaires actuellement en vigueur concernant les infrastructures et les contenus. Il s'agirait, au minimum, de procéder à un réexamen et à un amendement d'accords tels que l'Accord mondial sur les télécommunications, le système international de compensation des revenus des télécommunications internationales et l'Accord sur les aspects des droits de propriété intellectuelle qui touchent au commerce (ADPIC).

\section{L’Organisation mondiale du commerce (OMC)}

Les accords internationaux actuellement en vigueur dans le domaine des télécommunications sont inspirés, dans une large mesure, par l'idée selon laquelle

\footnotetext{
PNUD, Rapport mondial sur le développement humain, Oxford, 1998, p. 37.

PNUD, op. cit.

UIT, Rapport sur le développement des télécommunications dans le monde. Echanges commerciaux dans le domaine des télécommunications, Genève, 1997.
} 
l'infrastructure de télécommunications d'un pays peut être administrée par des sociétés privées et que, lorsque des parties du réseau ne sont pas rentables, l'Etat peut fournir au public des moyens de faire en sorte qu'aucun citoyen ne soit exclu. A l'occasion du $2^{\mathrm{e}}$ Forum mondial des politiques de télécommunication, consacré au commerce des télécommunications, organisé à Genève du 16 au 18 mars 1998, le secrétaire général de l'UIT de l'époque, Pekka Tarjanne, a insisté sur les principaux éléments de la gouvernance mondiale en matière de télécommunications. Selon Tarjanne, le monde des télécommunications a profondément changé avec l'adoption de l'Accord de l'OMC sur les services de télécommunications de base et de l'Accord général sur le commerce des services (AGCS), qui l'avait précédé.

L'industrie des télécommunications fonctionne maintenant, à tous égards, dans un cadre commercial. Dans la transition vers un environnement axé sur le marché, il est important que tous les pays puissent tirer pleinement parti des nouvelles possibilités que créera un marché mondial des télécommunications libéralisé et dynamique.

L'accord qui transforme ces principes en droit contraignant est l'Accord mondial sur les télécommunications. L'Accord de Marrakech instituant l'Organisation mondiale du commerce (15 avril 1994) constitue l'acte final du Cycle de négociations de l'Uruguay (le huitième cycle de négociations commerciales multilatérales tenues sous les auspices du GATT). Le traité final contient un Accord général sur le commerce des services (AGCS). L'annexe la plus élaborée concerne les télécommunications. Elle définit de la manière suivante le service et le réseau publics de transport des télécommunications:

- Service public de transport des télécommunications: tout service de transport des télécommunications qu'un Membre oblige, expressément ou de fait, à offrir au public en général.

$\square$ Réseau public de transport des télécommunications: l'infrastructure publique de télécommunication qui permet les télécommunications entre deux extrémités terminales définies du réseau ou plus.

Parmi les 125 pays signataires de l'Accord de Marrakech, une soixantaine se sont engagés à ouvrir leur marché aux services de télécommunications, bien que la plupart ne se soient pas engagés sur la question des télécommunications de base. Les engagements vont de l'ouverture totale à la concurrence pour tous les services de télécommunications à des exceptions pour les services de télécommunications de base, les services cellulaires ou les services locaux. La réunion de Marrakech a créé le Groupe de négociation sur les télécommunications de base (GNTB), chargé de traiter des services de télécommunications et censé achever ses travaux avant avril 1996.

Le GNTB n'est pas parvenu à conclure un accord à la date fixée. Plusieurs questions n'ont pu être réglées, comme la libéralisation des services par satellite et les arrangements de compensation pour les tarifs des télécommunications internationales. Les négociations ont cependant abouti à un accord sur certaines règles de base, formulées dans un texte dit «document de référence», qui traite 
des sauvegardes en matière de concurrence, de l'interconnexion, des obligations relatives au service universel, de la transparence des critères d'attribution des licences, de l'indépendance du régulateur et de l'attribution et de l'utilisation des ressources limitées.

En juillet 1996, un nouveau groupe - le Groupe des télécommunications de base - a pris la relève. Ouvert à tous les Etats membres de l'OMC, il tenait des réunions mensuelles. Sa tâche principale était d'encourager davantage de pays à prendre des engagements, de traiter de la question de la libéralisation des services par satellite et de régler un certain nombre de problèmes liés à l'offre de services de télécommunications.

Les nouvelles négociations ont porté, entre autres, sur la question des restrictions à la participation étrangère au capital des entreprises de télécommunication. Le gouvernement des Etats-Unis a fortement pesé pour que soit autorisée une participation étrangère maximale dans les sociétés de télécommunication nationales. En formulant leurs engagements, de nombreux pays ont levé leurs restrictions aux prises de participation étrangères, tandis que d'autres ont maintenu une part de contrôle national allant de $25 \%$ à $80 \%$. Alors que certains pays voient dans les participations étrangères une possibilité d'attirer les investissements étrangers dont ils ont besoin ${ }^{8}$, d'autres y voient une menace contre leur souveraineté nationale. Les gouvernements nationaux ont la maîtrise complète de l'ampleur, du calendrier et du moment de leurs engagements. Toutefois, une fois qu'ils ont pris ces engagements, ils ne peuvent plus par la suite revenir sur leurs concessions.

Le 15 février 1997, 72 Etats membres de l'OMC (qui représentaient environ 93\% du commerce mondial de services de télécommunications) signaient le quatrième protocole annexé à l'Accord général sur le commerce des services. Le 5 février 1998, ce protocole est entré en vigueur. Cet Accord mondial sur les télécommunications impose aux Etats participants de libéraliser leur marché. Ils disposent d'une certaine souplesse pour mettre en œuvre l'accès universel d'une manière qui leur convienne, mais l'accord contient des conditions sévères qui restreignent fortement l'espace de manœuvre politique national.

L'accord a des conséquences très importantes pour la gestion des infrastructures de base en matière de télécommunications. C'est ainsi qu'il affirme, au sujet du service universel: «Tout membre a le droit de définir le type d'obligation en matière de service universel qu'il souhaite maintenir. Ces obligations ne seront pas considérées comme étant anticoncurrentielles en soi, à condition qu'elles soient administrées de manière transparente, non discriminatoire et neutre du point de vue de la concurrence et qu'elles ne soient pas plus rigoureuses qu'il n'est nécessaire pour le type de service universel défini par le membre.» Avec cette disposition, les signataires ont accepté que les conditions locales en matière d'accès universel ne soient pas «plus rigoureuses qu'il n'est nécessaire». Voilà qui limite grandement la marge de manœuvre d'une politique nationale sur la question de l'accès. Comme les industries étrangères ne peuvent

$8 \quad$ Ibid., p. 102. 
être placées en situation défavorable, les critères nationaux pour les normes du service universel doivent être administrés de manière neutre du point de vue de la concurrence. Ils ne peuvent être fixés à des niveaux «plus rigoureux que nécessaire». Si une politique publique nationale envisageait d'offrir un accès aux services de télécommunications sur la base d'un système de financement croisé plutôt que sur la base de tarifs fondés sur les coûts, cela pourrait répondre davantage aux besoins des petits usagers qu'à ceux des opérateurs des télécommunications; en pareil cas, les acteurs étrangers verraient très probablement cette obligation comme «plus rigoureuse qu'il n'est nécessaire». Par voie de conséquence, une telle politique serait considérée comme contraire au droit commercial international. Il reviendrait aux mécanismes d'arbitrage (qui ne brillent pas par leur clarté) de juger du caractère légitime ou illégitime de la proposition de politique nationale.

L'accord insiste davantage sur l'accès que les fournisseurs étrangers devraient avoir aux marchés nationaux des télécommunications que sur l'accès des citoyens nationaux à l'utilisation des services de télécommunications. C'est faire preuve de simplisme que d'assimiler ces formes différentes d'accès.

La conséquence en est que la politique sociale est confinée dans des limites fixées par les acteurs commerciaux. Ce sont les intérêts commerciaux, plutôt que les aspirations socioculturelles, qui déterminent les politiques nationales des télécommunications. Conformément à l'accord, l'OMC a suggéré que le marché des télécommunications de base soit entièrement ouvert d'ici à 2004, dans le monde entier ou presque (probablement à hauteur de $93 \%$ ), puisque la plupart des partenaires commerciaux ont accepté de libéraliser leur marché intérieur. La création de marchés libres à l'échelle mondiale pour tous les types de services ne signifie cependant pas nécessairement que ces services soient disponibles, ni que toutes les personnes susceptibles d'en bénéficier puissent les utiliser de manière équitable.

Si l'on en juge par la participation croissante aux négociations sur les télécommunications et par la multiplication des engagements d'ouverture des marchés, force est de conclure qu'un nombre croissant de pays considèrent que la libéralisation de leur marché des télécommunications leur apportera des avantages. Le véritable enjeu politique n'est plus de savoir si les pays vont libéraliser ou non, mais plutôt à quel moment ils le feront. Pourtant, les opinions divergent toujours, comme le relève le Rapport sur le développement des télécommunications dans le monde de l'UIT: «L'accès aux marchés, par exemple, sera considéré par certains comme une chance à saisir, tandis que d'autres, qui s'efforcent de développer leur propre industrie nationale de services de télécommunications, pourraient y voir un défi et une menace contre des opérateurs locaux balbutiants. ${ }^{9}$

La libéralisation entraînera dans certains pays une augmentation des recettes pour les opérateurs nationaux, tandis que dans d'autres pays la majeure partie des revenus pourrait aller à des entités étrangères. Comme le relève fort justement le rapport de l'UIT, «il y aura des gagnants et des perdants ${ }^{10}$.

9 Ibid.

$10 \quad$ Ibid., p. 106. 


\section{Privatisations en cascade}

Dans le cadre de l'ouverture de leurs marchés, bien des pays ont aussi entrepris de privatiser leurs opérateurs publics de télécommunications. Depuis 1984, 44 opérateurs publics ont été privatisés ${ }^{11}$. Ces privatisations ont rapporté quelque 159 milliards de dollars. Les 12 plus grandes privatisations de 1996 ont permis de lever plus de 20 milliards. Les investissements étaient, grosso modo, nationaux à raison de $50 \%$ et étrangers pour $50 \%$. De manière générale, plus de $30 \%$ des capitaux investis étaient de source étrangère. Comme l'indique l'UIT, les opérateurs publics eux-mêmes sont généralement les investisseurs les plus actifs. Toutefois, en 1997, les Etats détenaient toujours des participations majoritaires dans 29 des 40 principaux opérateurs internationaux. «C'est moins une privatisation complète qu'une transformation en sociétés privées de compagnies publiques de télécommunications qui a gagné toutes les régions du monde. $\gg^{12}$ En outre, la libéralisation n'a pas abouti à la création de marchés concurrentiels dans l'ensemble des secteurs dans tous les pays. Dans de nombreux pays, les services de télécommunications de base ne sont pas ouverts à la concurrence. Certains marchés de téléphonie mobile figurent parmi les plus libéralisés, mais même dans ce secteur, plusieurs pays n'autorisent pas (encore) la concurrence.

Les partisans de la privatisation évoquent volontiers l'expansion et la mise à niveau des réseaux, l'amélioration des services et la réduction des tarifs pour l'accès aux réseaux et leur utilisation. Or, les enseignements tirés de l'expérience sont mitigés. Dans plusieurs pays, les prix ont bien baissé, mais avant tout pour les grosses entreprises; l'usager moyen n'a guère vu baisser ses factures de téléphone. En matière d'offre de services, les expériences sont aussi assez diverses. Cela s'explique en partie par le fait que les attentes d'une concurrence accrue et d'un choix élargi grâce à la privatisation n'ont pas toujours été remplies. Il se trouve que dans des pays plus petits et moins avancés, les opérateurs nationaux de télécommunications ont perdu la partie contre les grandes coalitions planétaires - les nouveaux monopolistes. Le consommateur a-t-il quelque chose à gagner lorsque les marchés sont aux mains d'un petit nombre d'opérateurs mondiaux? Il est permis d'en douter, de même que l'on peut se demander quelle sera la concurrence qui survivra en définitive, étant donné que la baisse des prix et des investissements pour l'innovation technologique tend à évincer les concurrents du marché, et que la libéralisation des marchés conduit presque partout à une concentration accrue.

La croyance la plus curieuse est cette idée répandue que la libéralisation des marchés crée nécessairement un marché concurrentiel. L'histoire enseigne pourtant que les marchés libres mènent inévitablement à la constitution de monopoles, puisque les concurrents évincent les prétendants du marché ou fusionnent avec eux. La vraie question n'est pas l'alternative entre monopole ou concurrence, mais plutôt l'efficacité comparée des monopoles d'Etat et des monopoles commerciaux.

\footnotetext{
Ibid., p. 2.

12 UIT, Tendances générales des réformes dans les télécommunications, vol. 1, Mondial, $1^{\text {re }}$ édition, Genève, 1998, p. 9.
} 
La privatisation a souvent pour effet l'expansion du réseau des télécommunications. Dans plusieurs pays (comme, en 1997, le Pérou et le Panamá), la privatisation a fortement densifié le réseau. Selon l'UIT, «l'une des raisons est que les objectifs d'expansion des réseaux sont de plus en plus souvent intégrés dans les concessions de privatisation $»^{13}$. Les nouvelles lignes téléphoniques sont bien entendu bénéfiques pour les usagers qui peuvent se permettre ce service. La privatisation n'élargit pas le cercle des citoyens dotés du pouvoir d'achat nécessaire pour utiliser les réseaux de télécommunications.

C'est toujours le volume des communications internationales qui permet à l'opérateur national de télécommunications de dégager des bénéfices. Pourtant, les gains financiers obtenus grâce aux services de communication avec l'étranger ne bénéficient pas nécessairement à l'opérateur national. Bien des pays décident, pour des raisons politiques et autres, de ne pas réinvestir une proportion suffisante de ces recettes dans l'expansion et la mise à niveau des installations de télécommunications. Qui plus est, la concurrence est de plus en plus vive sur le marché des services de communication internationaux, et les tarifs sur les liaisons les plus fréquentées subissent une forte pression à la baisse.

\section{La répartition des recettes}

La disponibilité de financements pour l'expansion et l'amélioration des infrastructures de télécommunications dépend aussi d'accords internationaux relatifs à la répartition des recettes de la téléphonie internationale. L'une des raisons d'être fondamentales de la réglementation des télécommunications, telle qu'elle fut consacrée par la première Convention internationale des télégraphes (UIT, 1865), était de trouver un système adéquat pour le partage des revenus des appels internationaux entre pays d'origine, de transit et de destination. Grosso modo, l'opérateur public de télécommunications dans le pays d'origine facturait un certain prix au client; les opérateurs publics du pays de destination et du pays d'origine s'entendaient ensuite sur un prix pour les services fournis par l'opérateur du pays de destination (fourniture de lignes internationales, commutation et acheminement des appels aux clients locaux). La somme en question formait la base des tarifs facturés par l'opérateur du pays de destination à l'opérateur du pays d'origine. Ces tarifs sont appelés «taxes de répartition». La recommandation générale de l'UIT consistait à diviser les frais en deux parts égales entre les opérateurs. Ce système fonctionnait bien dans une situation de monopole à monopole, où les télécommunications internationales étaient considérées comme un service offert conjointement. Le tableau change du tout au tout avec l'arrivée d'un plus grand nombre d'opérateurs privés, avec une concurrence accrue et avec des options techniques qui permettent de contourner le système existant.

Voici déjà un certain temps qu'une réforme du système actuel de clés de répartition est débattue, au sein de l'Organisation de coopération et de développement économiques (OCDE) depuis 1991, à l'UIT depuis 1992, et à l'OMC. Par le passé, le système existant a bien servi les intérêts des pays en développement.

13 Ibid., p. 71. 
Comme ils appliquaient généralement des tarifs assez élevés pour assurer l'acheminement sur leur territoire des appels internationaux, le système de répartition des recettes était pour eux une source importante de devises étrangères. Ces recettes peuvent - en principe tout au moins - être utilisées pour favoriser l'accès aux infrastructures de télécommunications pour les habitants des zones rurales qui sans cela demeureraient à l'écart.

Selon l'UIT, les pays en développement perçoivent à ce titre des paiements nets qui représentent jusqu'à 10 milliards de dollars par an. Comme les négociations sur la réforme du système ne progressaient pas assez vite, le gouvernement des Etats-Unis a décidé d'annoncer unilatéralement la solution qui avait sa préférence. La Federal Communications Commission (Commission fédérale des communications, FCC) a déclaré que les Etats-Unis perdaient ainsi chaque année des milliards de dollars sous forme de paiements à d'autres pays. Elle a donc introduit, en novembre 1996, un avis de projet de réglementation qui est entré en vigueur en janvier 1998, instaurant un système révisé qui définit les sommes que les opérateurs des Etats-Unis peuvent verser aux opérateurs des pays étrangers (ces sommes représentent en moyenne la moitié des montants versés par le passé).

Dans le contexte de ses politiques de libéralisation et d'encouragement de la concurrence, l'Union européenne a décidé de cesser d'appliquer le système des taxes de répartition pour les télécommunications entre les pays membres. L'Union préfère appliquer un système de taxes basées sur les coûts pour les services d'interconnexion.

Les critiques du système classique prétendent que des paiements supérieurs aux coûts réellement encourus ne sont plus appropriés dans un marché des télécommunications qui fonctionne de plus en plus selon un modèle commercial. Ils avancent qu'il est inacceptable pour les pays d'origine de verser des sommes à tel point supérieures aux coûts d'acheminement de leurs appels, et que les pays de destination peuvent subventionner leurs politiques d'accès universel. Les défenseurs du système évoquent, quant à eux, l'importance vitale de ces paiements pour instaurer un accès universel dans les pays pauvres, et affirment que la baisse des paiements au titre des taxes de répartition conduira inéluctablement à une hausse des coûts pour les clients locaux dans leurs pays. La forme que prendra en définitive le système de gestion des taxes aura un impact très lourd non seulement sur des questions telles que l'accessibilité des TIC dans les pays les plus pauvres, mais aussi sur la situation économique générale de ces pays.

\section{Les droits de propriété intellectuelle}

Il existe encore un arrangement juridique international de première importance: celui qui concerne la protection des droits de propriété intellectuelle. Dès le départ, la protection des droits de propriété intellectuelle reposait sur trois idées fondamentales. La première était que les personnes qui ont investi pour produire la propriété intellectuelle devaient se voir garantir une rémunération financière. Lors de la rédaction des premiers traités internationaux sur la protection de la propriété intellectuelle (la Convention de Paris de 1883 pour la protection de la 
propriété industrielle et la Convention de Berne de 1886 pour la protection des œuvres littéraires et artistiques), la rémunération du créateur fut considérée comme une mesure d'incitation nécessaire pour encourager les investissements dans l'innovation et la créativité. Lors de la révision de la Convention de Berne, en 1928, la notion de droit moral fut ajoutée à celle du droit à rémunération. L'introduction de la valeur morale des œuvres reconnaissait le fait que celles-ci représentaient la personnalité intellectuelle de leur auteur. Les droits moraux protègent l'œuvre de création contre toute modification apportée sans le consentement de l'auteur; ils protègent le statut de celui-ci en tant qu'auteur de l'œuvre et son droit de décider de la publication.

L'intérêt public de la protection de la propriété intellectuelle est une notion qui est apparue assez tôt dans l'histoire du développement du droit de la propriété intellectuelle. Le principe général selon lequel les droits de propriété intellectuelle, en favorisant l'innovation et le progrès dans les domaines artistique, technologique et scientifique, contribuent par là au bien-être public a été reconnu. La protection de la propriété intellectuelle constitue donc en réalité un équilibre délicat entre des intérêts économiques privés, la propriété individuelle, des valeurs morales et des intérêts publics. Au cours des dernières années, le régime international de protection de ces droits s'est écarté de la dimension morale et de l'intérêt public, penchant davantage, dans sa pratique concrète, vers les intérêts économiques des titulaires de droits de propriété intellectuelle. Aujourd'hui, ces titulaires ne sont plus, la plupart du temps, des auteurs et des compositeurs qui créent des produits culturels, mais des sociétés transnationales productrices. Ces acteurs sont bien plus préoccupés par leur lutte contre le «piratage» et les pertes potentielles qu'il peut leur infliger que par la protection de l'intégrité morale d'œuvres de création ou par la qualité de la vie culturelle dans le monde.

L'Accord sur les aspects des droits de propriété intellectuelle qui touchent au commerce (ADPIC), conclu dans le cadre des négociations du GATT (sous forme d'annexe 1C à l'Accord général sur les tarifs et le commerce dans le cycle des négociations commerciales multilatérales de l'Uruguay de 1993), est désormais appliqué dans le cadre réglementaire de l'OMC. Dans cet accord, c'est la dimension économique de la protection des droits de propriété intellectuelle qui est renforcée. Shalini Venturelli résume bien la situation lorsqu'elle écrit: «La balance penche désormais entièrement du côté des intérêts des tiers exploitants en matière d'incitation économique, au détriment des intérêts de l'accès public des citoyens et des droits constitutionnels et humains du travail de création. ${ }^{14}$ Comme les droits de propriété intellectuelle occupent désormais une place de choix parmi les biens commercialisables les plus importants du monde, le régime actuel de ces droits, orienté vers le commerce, favorise les sociétés productrices (éditeurs, sociétés de radiodiffusion, compagnies d'enregistrement de musique, sociétés de publicité) aux dépens des créateurs. Les dispositions de l'Accord sur les ADPIC sauvegardent les droits économiques des investisseurs bien mieux que les droits moraux des créateurs individuels ou les intérêts culturels du grand public.

14 Venturelli, S., «Cultural Rights and World Trade Agreements in the Information Society», Gazette, vol. $1, n^{\circ} 60,1998$, pp. 47-76, cit. p. 63. 
Cette tendance à la protection des intérêts économiques des producteurs commerciaux transparaissait clairement dans les tentatives du gouvernement des Etats-Unis et de l'Union européenne d'inclure les bases de données numériques et les copies numériques (même temporaires) dans la révision de la Convention de Berne, à la fin de 1996. A l'appui de cette position, un groupe de pression puissant s'est constitué, combinant les ressources et les intérêts de la Motion Pictures Association, de la Fédération internationale de l'industrie phonographique (IFPI), de la Federation of European Publishers et de la Business Software Alliance, regroupant des sociétés comme Microsoft, Apple et IBM. Ces groupes ont proposé l'inclusion dans l'article 7 du traité révisé d'une disposition selon laquelle «la reproduction directe et indirecte de ces œuvres, qu'elles soient permanentes ou temporaires, de quelque manière ou sous quelque forme que ce soit», tomberait sous le coup de la protection du droit d'auteur. Pour la consultation de sources sur Internet, cela reviendrait à un système de "paiement à la séance». Les opposants à cette proposition (un groupe de pression qui réunissait des sociétés de télécommunications - AT\&T, Philips, British Telecom et France Telecom -, la société Netscape, qui produit un logiciel de navigation sur le web, des bibliothèques, des fournisseurs d'accès à Internet et des usagers d'Internet) affirmaient quant à eux que l'article 7 ainsi rédigé saperait l'attrait d'Internet et entraverait gravement l'accès gratuit à l'information.

Le 20 décembre 1997, la conférence diplomatique décidait de supprimer l'article 7 si contesté. Cette mesure mettait un terme, provisoirement au moins, à l'extension de la protection du droit d'auteur, mais il n'est pas sûr que les tenants de cette option s'arrêteront là. La directive CEE du 11 mars 1996 concernant la protection juridique des bases de données montre que la tentative d'étendre la protection du droit d'auteur aux sources d'information numériques se poursuit sans relâche. La directive soumet l'accès gratuit aux banques de données aux dispositions relatives au droit d'auteur.

L'argument clé en faveur du renforcement de la protection des droits de propriété intellectuelle (entre autres par l'expansion de ces droits et par la répression sévère des infractions) est généralement inspiré par les pertes (qui se chiffrent en milliards de dollars) que les industries culturelles subissent du fait du piratage à l'échelle mondiale. Les sociétés américaines de logiciels, les entreprises de loisir et les éditeurs affirment avoir perdu quelque 14 milliards de dollars en 1995 en raison des agissements des pirates. La International Federation of Phonogram and Videogram Producers estime que près d'un quart de tous les enregistrements musicaux sont des copies pirates. L'un des problèmes difficiles que pose la tendance actuelle en matière de protection des droits de propriété intellectuelle est le fait que le cadre réglementaire qui se constitue compromet l'indépendance et la diversité de la production créative partout dans le monde. Le régime est particulièrement défavorable pour la protection des «petits» auteurs indépendants de produits créatifs.

Le système crée des obstacles formidables à l'utilisation de produits de création, car il limite la notion d' «usage équitable» permettant traditionnellement à ces produits d'être utilisés librement pour une diversité d'usages, parmi lesquels l'éducation. Son point de vue étroitement économique vise davantage le vol de 
biens appartenant à des entreprises privées que l'innovation et la création artistique et littéraire. Phénomène particulièrement inquiétant, le régime prévoit que les produits culturels relevant du «domaine public», lorsqu'ils sont placés dans des bases de données électroniques, tombent sous le coup de la protection des droits de propriété intellectuelle. Il en découlera une restriction considérable des possibilités d'accès à des sources libres de droits. Qui plus est, le régime menace de faire du nouveau forum global que le cyberespace pourrait offrir (grâce aux NTIC) un marché sur lequel un volume limité d'idées sera commercialisé.

\section{Et le code de la CNUCED?}

En plus de réexaminer et d'amender les arrangements internationaux évoqués ci-dessus, il serait essentiel pour la communauté internationale d'adopter enfin le projet de Code international de conduite pour le transfert de technologies, élaboré dans le cadre de la Conférence des Nations unies pour le commerce et le développement (CNUCED). Depuis 1977, plusieurs versions du projet de code ont été discutées, mais les négociations n'ont toujours pas abouti. L'objet principal du code est d'instituer un arrangement équitable permettant aux pays les plus pauvres de bénéficier eux aussi des progrès techniques et de commencer à maîtriser leurs propres processus d'innovation technologique. Le principal obstacle empêchant de parvenir à un accord en matière de transfert de technologies est le fait que le groupe puissant de sociétés fournisseuses de technologies ne voit aucun intérêt à créer des conditions permettant aux pays du tiers-monde d'acquérir des connaissances techniques et de contrer les pratiques commerciales agressives des sociétés transnationales propriétaires des technologies.

\section{Vision et pouvoir}

Etant donné les réalités politiques et économiques actuelles, il est vraisemblable que le SMSI finira par être le théâtre de l'affrontement auquel nous n'avons déjà assisté que trop souvent, entre vision et pouvoir. Il n'y a rien, dans toutes les belles déclarations préparatoires, qui pourrait amener à penser que la vision d'avenir aura cette fois le dessus sur le pouvoir. Ce sont en définitive les relations de pouvoir dans le monde qui détermineront si les visions d'avenir proposées ont une chance réaliste d'être traduites en actes. Il faut bien comprendre que dans le contexte actuel de la politique mondiale, le pouvoir d'arrêter les grandes orientations est essentiellement aux mains d'un petit nombre d'institutions gouvernementales (comme l'Organisation mondiale du commerce) et d'initiatives privées (comme le Global Business Dialogue on Electronic Commerce).

Au cours des dix dernières années, la politique mondiale de la communication a été le théâtre de bouleversements majeurs. On peut citer, parmi les facteurs essentiels, les points suivants.

$\square$ Le système international de gouvernance de la communication en place depuis un siècle servait avant tout à coordonner des politiques nationales formulées de manière indépendante par des gouvernements souverains. Le système actuel de gouvernance globale détermine dans une large mesure à l'échelle supranationale l'espace dont disposent les gouvernements nationaux pour fixer leurs politiques nationales. 
$\square$ La politique mondiale de la communication est de plus en plus déterminée par les critères du commerce et du marché, et de moins en moins par des considérations d'ordre politique. On constate un passage indéniable d'un discours à prédominance politique à un discours dominé par l'économie et le commerce. Pour preuve, l'insistance de plus en plus marquée sur l'importance économique de la propriété intellectuelle et sur la priorité qui doit, de ce fait, être accordée à la protection des investisseurs et des entreprises productrices. Dans le secteur des télécommunications, les normes du service public universel et du financement croisé ont cédé la place à des structures tarifaires basées sur les coûts. Dans le secteur des flux de données électroniques transfrontières, le débat politique ne porte plus sur la souveraineté nationale ni sur l'autonomie culturelle, mais sur les obstacles au commerce et sur l'accès aux marchés.

$\square$ Les acteurs privés les plus puissants ont acquis un poids prépondérant. La «main invisible» des intérêts économiques - qui ont toujours guidé les décisions politiques - s'est faite de plus en plus visible depuis quelques années. Les entreprises transnationales sont devenues des acteurs de premier plan, qui remplissent leur rôle de la manière la plus explicite sous les feux de la rampe. En vérité, le processus de définition des politiques a passé des gouvernements à des associations d'acteurs de l'entreprise privée. On peut citer comme exemple récent d'association de ce type le Global Business Dialogue on Electronic Commerce, créé en 1998 par des sociétés du secteur de l'information et des communications pour traiter de questions réglementaires dans le domaine de l'Internet, et en particulier du commerce électronique.

$\checkmark$ La politique mondiale de la communication était habituellement définie dans des instances intergouvernementales telles que l'UNESCO, l'Organisation mondiale de la propriété intellectuelle et l'Union internationale des télécommunications. Ces organisations étaient relativement ouvertes à la dimension socioculturelle des évolutions dans le domaine des TIC. Qui plus est, elles offraient une tribune à laquelle les intérêts des pays en développement pouvaient aussi être défendus. Depuis quelques années, la position de ces organisations intergouvernementales a été considérablement affaiblie, dans la mesure où les principaux acteurs ont commencé à leur préférer une instance plus favorable à leurs intérêts spécifiques. Cette instance est le successeur de l'Accord général sur les tarifs douaniers et le commerce: il s'agit de l'Organisation mondiale du commerce (OMC). La création de l'OMC est l'un des résultats de la conclusion du cycle de négociations commerciales multilatérales dit de l'Uruguay, qui s'est achevé en décembre 1993. L'OMC est, de manière générale, plus favorable aux intérêts commerciaux des grands pays industrialisés que les autres organismes intergouvernementaux, et ce bien que ses grands principes politiques soient la libéralisation des marchés dans le monde entier, le principe de non-discrimination qui prévoit que les concurrents étrangers doivent bénéficier sur le marché intérieur du traitement national, et le traitement de la nation la plus favorisée.

A vrai dire, on ne saurait s'étonner de ce que la politique de la communication ait désormais déplacé son centre de gravité vers cette organisation vouée au commerce, étant donné la valeur économique croissante des réseaux de commu- 
nication et des services d'information. Le marché mondial des communications pèse aujourd'hui près de 3 billions de dollars par an. Si l'on ajoute à cela le fait que les grandes sociétés de communication et d'information fournissent les structures d'appui essentielles aux marchés des matières premières et aux marchés financiers, on conçoit que la gestion des secteurs d'activité liés à la communication soit désormais largement destinée à être soumise à un régime commercial globalisé.

La politique mondiale de la communication connaît donc une phase de transition historique; hier encore fondée sur le service public, elle passe à un régime axé sur la concurrence du secteur privé et sur des services de nature commerciale. C'est l'intérêt public qui risque fort d'être le perdant dans ce processus. La gestion globale de la communication et de l'information qui se met actuellement en place vise, pour une bonne part, à réduire au minimum l'intervention publique et à accroître le plus possible la liberté pour les forces du marché. Face aux grands centres de pouvoir dans la gouvernance mondiale de la communication, qui donnent la priorité aux conceptions de la société de l'information axées sur le marché, les conceptions centrées sur l'être humain sont défendues par des personnes et des organisations qui sont sincèrement convaincues de l'importance de ces aspirations et qui estiment que les innovations dans le domaine des TIC ouvrent des possibilités nouvelles pour leur réalisation. Mais ces propositions sont aussi formulées, dans certains cas, par des particuliers et des institutions qui utilisent ces visions comme des écrans de fumée pratiques pour faire croire à une volonté de changement social qui n'a pas d'existence réelle. En un mot, les visions de la société de l'information que l'on trouve dans les textes préparatoires du SMSI sont inspirées soit par une grande naïveté, soit par une bonne dose de duplicité.

Que doivent penser les habitants de notre planète d'une nouvelle grand-messe internationale prétentieuse et coûteuse, qui ne remédiera en rien à leurs difficultés de tous les jours? Ce sommet ne fera qu'apporter de l'eau au moulin de la défiance croissante qu'éprouvent les peuples, dans le monde entier, à l'égard de la capacité des institutions politiques de représenter leurs intérêts.

Malgré toutes les phrases prometteuses des documents du SMSI, nous assistons en réalité à la mise en place «par le haut» d'une société globale de l'information qui se caractérise par les traits suivants:

$\square$ les déplacements des citoyens sont surveillés en permanence par les organes de répression et de renseignement;

$\checkmark$ les données réunies sur les citoyens, y compris ceux qui ne sont pas suspectés de comportement criminel, sont conservées pendant des durées indéterminées;

$\checkmark \quad$ les droits de propriété intellectuelle accordés aux entreprises sont indéfiniment étendus;

- l'accès à l'information et au savoir dépend de l'accès au pouvoir d'achat;

- la consolidation de la mainmise sur les marchés de l'information et des connaissances ne subit que des corrections mineures; 
$\square \quad$ il n'existe aucune restriction liée à la défense de l'environnement ou aux droits des travailleurs susceptible d'entraver la mise au point et l'application des TIC;

$\square$ davantage de personnes sont bien informées des pratiques de consommation que des pratiques politiques démocratiques;

$\square$ les entreprises privées qui détiennent la plupart des technologies et des contenus de la société de l'information n'ont que des obligations minimales de rendre compte publiquement de leurs actions;

$\square$ c'est le critère de la rentabilité, plus que celui de la sécurité humaine, qui guide le développement des TIC;

\lrcorner le domaine public est limité dans toute la mesure possible.

Toute tentative d'influencer le processus du SMSI dans le sens d'une vision axée sur l'être humain et les droits de l'homme exigerait de mobiliser un ensemble de partenaires sincèrement désireux de façonner une société de l'information à partir de la base. Un tel rassemblement pourrait être suscité parmi les organisations progressistes de la société civile, parmi les chercheurs en communication et en information qui mènent une réflexion critique, parmi les fonctionnaires qui ne se plient pas aux vues dominantes, parmi des représentants des milieux industriels et des ingénieurs faisant preuve d'un sens des responsabilités sociales, et parmi des travailleurs indépendants dans les domaines des médias, des télécommunications et de l'informatique.

Un tel groupe commencerait par effectuer l'analyse sociale fondamentale pemettant de mettre en lumière les obstacles à la mise en œuvre de projets par ailleurs louables. Dans un deuxième temps, il exercerait une pression publique sur les gouvernements pour que ces obstacles fassent l'objet de mesures sérieuses. Troisièmement, il refuserait d'être coopté par les gouvernements au sein de mécanismes ayant pour fonction essentielle de légitimiser des propositions d'action qui ne sont pas dans l'intérêt de la majorité de la population mondiale. Quatrièmement, il s'engagerait massivement dans des programmes d'éducation et dans des campagnes de mobilisation à l'intention du grand public, afin que les questions relatives à l'information et à la communication redeviennent des thèmes de débat social. Cinquièmement, il concevrait des exemples de meilleures pratiques de mesures «axées sur l'être humain» dans les domaines des médias, des télécommunications et de l'informatique. Sixièmement, il proposerait un programme politique inspiré par le respect des dispositions fondamentales des droits de l'homme inscrites dans le droit international. Septièmement, ce groupe proclamerait sa préoccupation à l'égard du système actuel de protection des droits de propriété intellectuelle, qui sanctionne le pillage à grande échelle des ressources d'information génétique des pays pauvres (biopiraterie) et qui protège les intérêts des sociétés propriétaires mieux que les intérêts des communautés locales ou des créateurs artistiques. Ce groupe serait intéressé au premier chef par la protection des intérêts de la propriété communale et des ressources culturelles, et par la protection des ressources tombées dans le domaine public contre leur exploitation par des sociétés privées. 
En matière de droits de l'homme, un groupe de ce genre plaiderait pour que la culture ne soit pas soumise aux dispositions commerciales et pour que des mesures soient autorisées à l'échelle nationale afin de protéger l'autonomie culturelle et l'espace public local. Les fusions qui se produisent actuellement à l'échelle mondiale ont des conséquences néfastes pour les droits de l'homme, aussi bien pour les consommateurs que pour les professionnels, en réduisant la diversité et en induisant une perte d'autonomie professionnelle. Il s'agirait, en matière de droits de l'homme, de préserver la propriété publique du patrimoine commun de l'humanité, afin que l'exercice de la responsabilité à l'égard du public et les exigences de la collectivité demeurent garantis.

Outre tout ce qui précède, le groupe évoqué ci-dessus recommanderait que la déclaration finale et le programme d'action du SMSI soient préfacés par la déclaration suivante en onze points, au nom des gouvernements qui adopteront ces textes:

- Nous estimons que les relations économiques qui régissent le monde d'aujourd'hui sont injustes au plus haut point et causent du tort à la majorité des habitants de la planète.

- Nous considérons que le processus actuel de mondialisation économique entraîne une répartition extrêmement inégale des avantages et des risques, dans le monde comme à l'intérieur des sociétés.

$\square$ Nous pensons que l'Organisation mondiale du commerce et ses politiques doivent subir une réforme radicale afin de répondre plus équitablement aux intérêts de la population mondiale.

- Nous considérons que le système des Nations unies doit être réformé afin de tenir pleinement compte des principes de participation démocratique et de responsabilité devant l'opinion.

$\square$ En ce qui concerne les armes de destruction massive, nous estimons que tous les pays doivent être désarmés.

$\square$ Nous refusons d'accepter plus longtemps que plus de 30'000 enfants meurent chaque jour de causes tout à fait évitables, à cause de la faim et d'autres facteurs dus à des défauts de l'ordre mondial actuel auxquels il est possible de remédier.

$\square$ Nous nous engageons en faveur d'une répartition plus équitable des ressources mondiales et, dans la mesure où nous venons de pays riches, nous ferons tout ce qui dépend de nous pour convaincre nos populations de modifier leur mode de vie consumériste.

$\square$ Nous entendons soutenir activement le fonctionnement efficace de la Cour pénale internationale à La Haye.

$\checkmark$ Nous nous engageons sans réserve pour le respect plein et entier de tous les droits de l'homme - civils, politiques, sociaux, économiques et culturels -, et nous instituerons des mécanismes plus efficaces pour leur défense.

$\checkmark$ Nous estimons que le noyau des arrangements démocratiques n'est autre que la confiance à l'égard du processus social, qui a été gravement ébranlée au cours des dernières années, et nous reconnaissons qu'il faut mettre un terme 
au recours courant, par les gouvernements, à des formes multiples de mensonge, de tromperie et de manipulation.

$\square$ Nous nous engageons à réexaminer, amender ou conclure tous les accords internationaux actuels (en vigueur ou en cours de négociation) traitant de questions relatives à la société de l'information, afin de garantir leur pleine conformité avec les dispositions du droit international relatives aux droits de l'homme.

Tout cela, j'en suis bien conscient, peut paraître terriblement romantique et utopique. Et pourtant, sans un engagement sincère sur de tels enjeux, toutes les belles paroles du SMSI resteront du vent pour la plupart des habitants de la planète. 